\title{
The Lithotripsy Table Height: a Novel Predictor of Outcome in Shockwave Lithotripsy
}

\author{
Enrique Ossandon, Pedro Recabal, Cristian Acevedo, Jose Miguel Flores, Fernando Marchant
}

Department of Urology, Hospital Clínico Universidad de Chile, Santiago, Chile

\begin{abstract}
Background: Outcome of Extracorporeal Shockwave Lithotripsy (SWL) is determined by physical factors that affect stone fragmentation and clearance.

Purpose: To evaluate the predictive value of the Lithotripsy Table Height (LTH) in SWL outcome. Lithotripsy Table Height (LTH) is a variable that represents skin to therapy head distance, and it is proportional to the energy that reaches the stone.

Materials and Methods: A prospective study enrolled patients undergoing SWL for radiopaque urinary stones. All procedures were performed using a Modulith SLX (Karl Storz, Germany) Lithotripter. Patient weight, height and age; stone location and size; number of shock waves delivered, and LTH were recorded. One month post-procedure a KUB was obtained. Logistic regression analysis was used to evaluate the effects of these variables on stone-free outcome. A ROC curve was plotted.

Results: Fifty-six patients were enrolled. After one month follow-up, overall success rate (Stone Free) was $83.9 \%(\mathrm{n}=$ 47). LTH was the only independent predictor of outcome in both univariate and multivariate analysis $(p=0.029)$. Stone size $(p=0.45)$ and BMI $(p=0.32)$ were not significant. In the ROC curve, LTH showed an Area under the Curve $=$ 0.791. Patients with $\mathrm{LTH}<218(\mathrm{n}=8)$ had relative risk of residual stones $=7.5$, odds Ratio: 6.6 (Stone free rate $37.5 \%$ vs. $91.5 \%)$.

Conclusion: LTH appears to be an independent predictor of SWL outcome. High success rates can be expected if LTH > 218. Patients with lower LTH had a less effective therapy, therefore, worse stone fragmentation and clearance. These findings may help improve patient selection for SWL therapy.
\end{abstract}

Key words: extracorporeal shockwave lithotripsy; high-energy shock waves; treatment outcome; calculi.

Int Braz J Urol. 2011; 37: 355-361

\section{INTRODUCTION}

Extracorporeal Shockwave Lithotripsy (SWL) represents a major technological breakthrough in the management of patients with stone disease. Since its introduction in the early eighties, millions of patients have benefited by this kind of therapy. In 1989 , a report by Chaussy (1), estimated that $70 \%$ of non selected urinary stone patients could be treated with SWL monotherapy, with an additional $25 \%$ able to be treated by SWL in combination with endourologic procedures. Differences between reported overall success rates for SWL range from 50 to $95 \%(2,3)$. Key factors that determine the outcome of patients undergoing SWL have been subject of intense research in recent years, the objective being a better selection of patients for this kind of therapy.

In this study, the predictive value of the Lithotripsy Table Height (LTH) in SWL outcome is evaluated. This variable is determined individually 
by the amount of tissue between the stone and the therapy head. Because both focal distance (distance from therapy head to the focal point; where pressure is maximum) and therapy head are fixed, to locate the stone in the focal point of the lithotripter, LTH is set by the operator for each patient before SWL begins, by moving the lithotripsy table along the $\mathrm{z}$ axis (up and down), using fluoroscopy at $30^{\circ}$, until the sum of Skin-to-Stone Distance (SSD) and Skin-to-Therapy Head Distance equal the focal distance. Since only Skin-to-Therapy Head Distance head is modifiable, LTH is directly proportional to the Skin-to-Therapy Head distance, and inversely proportional to SSD. Stones located deep within a patient's body (large skin-to-stone distance), require the table to be lower (i.e. closer to the therapy head) than superficial stones (small skin-to-stone distance; high table). It's important to note that this depends not only on the amount of fat, but also any other tissue interposed between the stone and the lithotripter, such as bone; connective tissue; renal parenchyma; etc., and location of the stone within the urinary system (for example, stones located in a calyx may be closer to the skin than one in the pelvis or ureter). The table's exact coordinate in the $\mathrm{z}$ axis is measured (in $\mathrm{mm}$ ) by a sensor in the SWL table, and displayed in real time in a digital screen, located on the control panel of the SWL machine. To provide appropriate conduction of the shock waves from the therapy head to human tissue, a cylindrical water cushion is interposed to fill the gap between the skin and the therapy head. This cushion is deflated to lower the table and inflated to elevate it. The LTH is proportional to the ratio of water (cushion) to tissue in the media where the shock waves are propagated, within a conical area with the focal distance as its height. If the table is high, the cushion is inflated and a large proportion of the conical area is water; if it's low, shockwaves are conducted mainly through human tissue (Figure-1). Our objective was to evaluate this variable as a predictor of stone fragmentation and clearance.

\section{MATERIALS AND METHODS}

A prospective cohort study to assess predictive value of LTH in SWL therapy was designed and

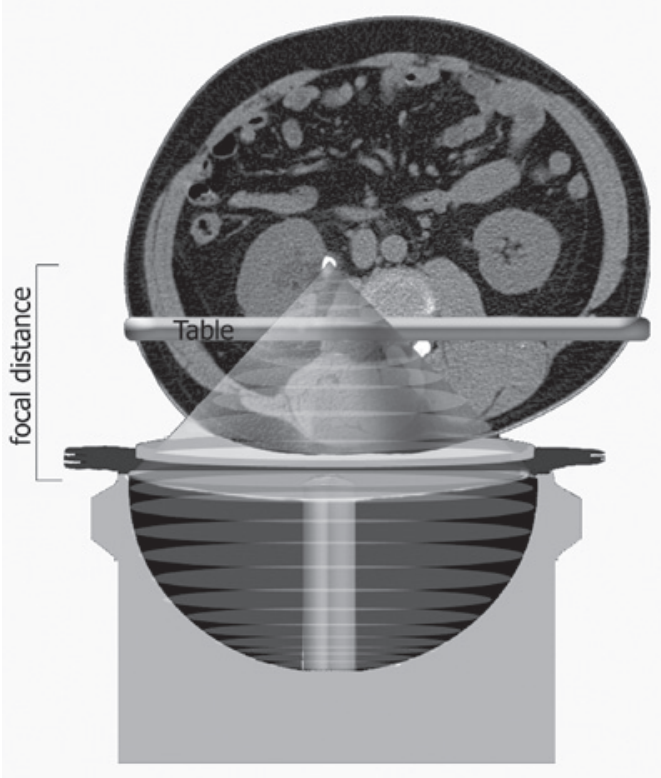

A

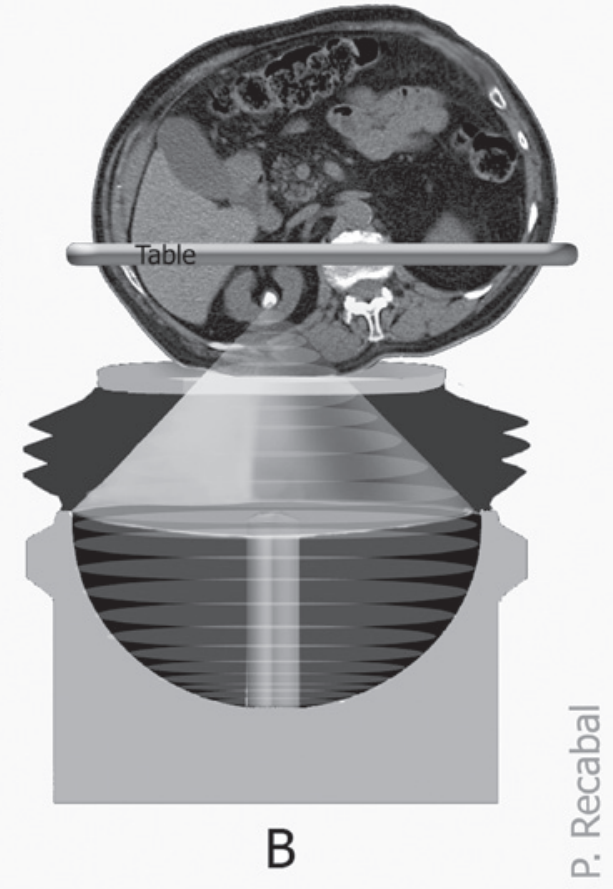

Figure 1 - If the table is low (A), tissue represents a large proportion of the media where shockwaves are being propagated, whereas when it is high (B), shockwaves are conducted mainly through water. 
conducted at Hospital Clínico Universidad de Chile. Calculated sample size was 50 patients, considering $\alpha=0.01$ power 0.9 , and Relative Risk $=5$. After approval by the review board, fifty-six untreated patients undergoing SWL at our institution were enrolled between October 2007 and July 2008. Inclusion criteria were having a solitary radiopaque urinary stone larger than $5 \mathrm{~mm}$ in its longest dimension as measured by non-contrast-enhanced computed tomography (NCCT) and SWL as the initial stone treatment. Written informed consent was obtained from each subject. SWL was done using a Modulith SLX (Karl Storz, Germany) Lithotripter in all procedures. This system unit is provided with a cylindrical electromagnetic shock wave source, X-ray C-arm for stone localization and patient table with floating table-top. By moving the patient table within a three dimensional Cartesian coordinate system under fluoroscopic guidance, stones were located on the focal point, $165 \mathrm{~mm}$ above the therapy head (focal distance for this lithotripter). LTH was set for each procedure by moving the table in along the $\mathrm{z}$ axis to locate the stone in the focal point, with an image intensifier at $30^{\circ}$ relative to the vertical. Each procedure was performed with the table at one particular coordinate in the $\mathrm{z}$ axis (Figure-1), which was recorded as each individual patient's LTH. Shock waves are delivered by means of externally-applied, high-intensity acoustic pulses, reflected off a parabolic reflector and focused above the therapy head at a fixed distance. All procedures were performed under conscious sedation. A maximum of 3000 shocks were delivered to kidney stones and 4000 shocks to ureteral stones or until the stone was completely fragmented. Shockwave frequency was $60 \mathrm{~min}-1$. Clinical data recorded included patient weight, height and age; stone location and size; number of shock waves delivered; and the LTH. A follow-up KUB was obtained at four weeks, and reviewed by a staff radiologist at our institution who was blinded to patient's body habitus and LTH. Stone free status was defined as absence of visible fragments on the KUB. Logistic regression multivariate analysis was used to evaluate the effect of several variables (LTH, BMI, stone size, number of shocks and age) on SWL outcome. A Receiver-Operating-Characteristic (ROC) curve was constructed to assess LTH test accuracy. A p-value of 0.01 or less was considered significant. Statistical analysis was performed using SPSS v15.0 and STATA 9.

\section{RESULTS}

Of the 56 patients, 39 were male and $17 \mathrm{fe}$ male. Stones were in the kidney in $25(44.5 \%)$ patients (of which 2 were lower pole stones) and in the ureter in $31(55.5 \%)$ patients. At one month follow-up, 47 $(83.9 \%)$ patients were stone free (absence of residual fragments); five had incomplete stone clearance, and four patients had no stone clearance $(7.1 \%)$ and had subsequent retreatment. The LTH ranged from 184 to 277. Eight out of nine patients with residual fragments had an LTH under the mean (235). The mean LTH in the stone free group was $239 \pm 17$ vs. $216 \pm 25$ in patients with residual fragments $(\mathrm{p}=0.001)$. Results are presented in Tables-1 and 2. In comparison of means

Table 1 - Clinical and demographic characteristics.

\begin{tabular}{lcccc}
\hline & $\begin{array}{c}\text { Overall Mean } \\
(\text { SD })\end{array}$ & $\begin{array}{c}\text { Stone Free Mean } \\
(\text { SD })\end{array}$ & $\begin{array}{c}\text { Residual Fragments Mean } \\
(\text { SD) }\end{array}$ & $p$-value \\
\hline $\mathrm{n}$ & 56 & 47 & 9 & \\
Stone size (mm) & $10.5(4.9)$ & $10.2(4.7)$ & $11.6(6.2)$ & 0.330 \\
BMI & $25.6(4.5)$ & $25.3(4.6)$ & $27.1(3.6)$ & 0.327 \\
LTH (mm) & $235(20.8)$ & $239.8(17.3)$ & $216.0(25.5)$ & $0.001^{*}$ \\
Number of Shocks & $2499(1015)$ & $2375.5(1002)$ & $3144.4(864)$ & $0.003^{*}$ \\
Age & $42.2(14.6)$ & $41.5(14.4)$ & $46.7(15.9)$ & 0.380 \\
\hline
\end{tabular}

Data for the entire group is presented as mean (standard deviation) in the second column. Data for Stone Free and Residual Fragment patients was analyzed by comparing means using t-test.

Patients with residual fragments present a lower mean LTH, and a higher BMI, stone size and Number of shocks. $/ S D=S t a n d a r d ~ D e v i a-$ tion. BMI = Body Mass Index. LTH = Lithotripsy Table Height. $/ *$ statistically significant $(p<0.05)$. 
The Lithotripsy Table Height

Table 2. Univariate and multivariate analysis of factors predicting residual fragments.

\begin{tabular}{lcccccc}
\hline \multicolumn{2}{c}{ Univariate Logistic regression } & & \multicolumn{3}{c}{ Multivariate Logistic regression } \\
\hline Variable & OR (95\% CI) & SE & p-value & OR (95\% CI) & SE & p-value \\
\hline Stone size & $0.95(0.84-1.08)$ & 0.06 & 0.451 & $0.96(0.81-1.15)$ & 0.03 & 0.663 \\
BMI & $0.93(0.81-1.07)$ & 0.07 & 0.324 & $1.02(0.84-1.23)$ & 0.10 & 0.880 \\
LTH & $1.06(1.02-1.11)$ & 0.02 & $0.002^{*}$ & $1.06(1.01-1.12)$ & 0.03 & $0.031^{*}$ \\
Age & $0.97(0.92-1.03)$ & 0.03 & 0.380 & $0.97(0.91-1.05)$ & 0.04 & 0.464 \\
$\mathrm{~N}^{\circ}$ of Shocks & $0.99(0.998-0.999)$ & 0.00 & $0.032^{*}$ & $0.99(0.998-1.000)$ & 0.00 & 0.277 \\
\hline
\end{tabular}

In the univariate analysis OR: Odds Ratios (95\% confidence intervals).

$S E=$ standard error. BMI = Body Mass Index. LTH = Lithotripsy Table Height. $/ *$ statistically significant.

using t-student, LTH and Number of shocks waves delivered were associated with outcome, where patients with residual fragments showed lower LTH and higher number of shocks delivered. No significant difference was found in LTH of patients in the supine vs. prone position ( $p=0.20)$, or if compared by side $(\mathrm{p}=0.24)$ or location (kidney vs. ureter; $\mathrm{p}$ $=0.26)$. No significant difference in outcome was noted when analyzed by stone location separating upper, mid and lower calyx, pelvis and upper mid and lower ureter $(\mathrm{p}=0.59)$, although X2 may be not be meaningful due to the low count in some cells. No significant difference in outcome was observed if analyzed by location as kidney vs. ureter, using Fisher exact test $(p=0.49)$. LTH was the only independent predictor of outcome in the multivariate analysis $(\mathrm{p}=0.02)$. A significant correlation was found between patient weight and LTH (Pearson $-0.41 ; p=0.002$ ), and between BMI and LTH (Pearson $-0.34 ; \mathrm{p}=0.010)$. In the ROC curve, the area under the curve was 0.79 for LTH (Figure-2). A cutoff LTH value of 218 yielded a sensitivity of $93.6 \%$, specificity of $55.5 \%$ and positive predictive value (PPV) of $91.5 \%$ for success. Patients with very low LTH (i.e. $<218 ; \mathrm{n}=8 ; 14.2 \%$ of the sample) had a SWL success rate of $37.5 \%$ for LTH $<218$ vs. $91.5 \%$ for LTH $>218$, and a Relative Risk of residual fragments $=7.5$.

\section{DISCUSSION}

In this study LTH appears as an independent predictor of outcome in SWL, showing statisti- cal significance in both univariate and multivariate analysis. This is, to our knowledge, the first report that addresses LTH as a predictor of outcome. Interestingly, the marked increase of residual fragments in patients with very low LTH (i.e $<218$ ), is noted in statistical analysis as the only variable with significant correlation to outcome, despite patients with residual fragments having received more shocks. BMI showed a significant correlation with LTH, but didn't reach statistical significance in the regression analysis. Accordingly, a study by Mensentzev (4) presented good SWL outcomes in morbidly obese patients, except when the stone was $>1 \mathrm{~cm}$ from the focal point. A tendency towards stone size was also noted. In our institution, patients with stones larger than $20 \mathrm{~mm}$ are primarily treated by endourological techniques, which explains the low number of stones larger than $20 \mathrm{~mm}$ in the cohort. For stones $20 \mathrm{~mm}$ or more $(n=4)$, both patients that were stone free had LTH above the media, whereas both patients with residual fragments had a LTH under the media. Apparently, this variable comes into consideration at the lower end of the distribution, as the energy delivered to the stone by means of the shockwave, is dissipated by reflection of part of the energy due to impedance mismatch in the media where it is being propagated (5), occurring not only when the shock wave propagates from water to tissue, but also within human tissue, due to different densities amongst different tissues such as skin, muscle, fat, kidney, etc... Thereby reducing the amount of energy that actually reaches the stone. This finding is a potential explanation to high SWL success rates in paediatric 


\section{ROC Curve}

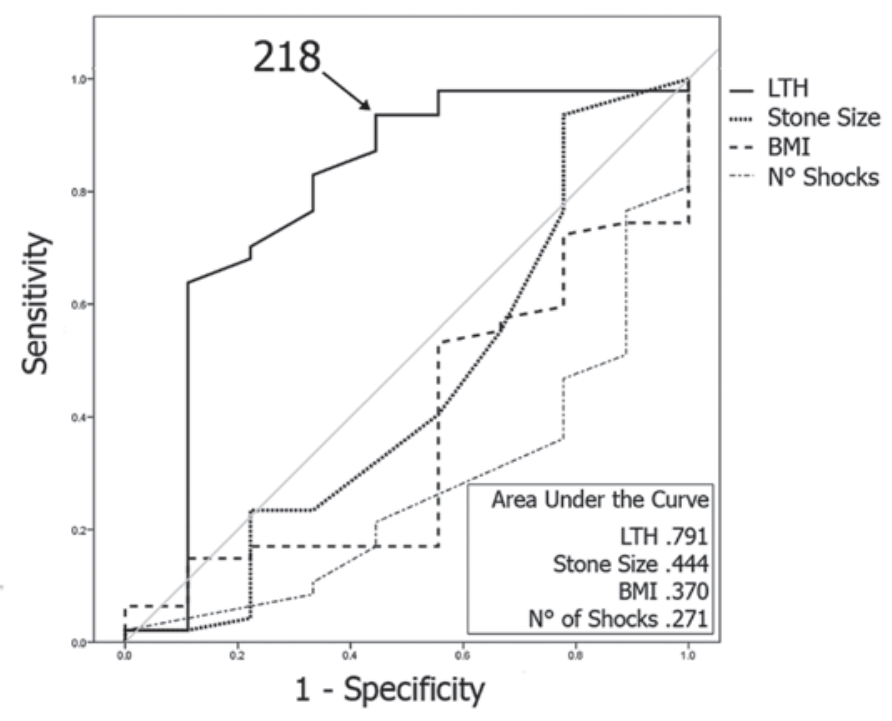

Figure 2 - Receiver operating characteristic curve.

patients (6-8), in which very high LTHs are to be expected. One drawback of this study is cohort size, as it was designed considering a large difference in means between groups, low statistical $\alpha$ value $=$ 0.01 , and potency $90 \%$, and is thereby underpowered to rule them out as predictors; if a smaller difference is to be detected, a larger cohort is required. Cohort size also impairs further analysis considering specific stone location within the urinary tract. Our study supports the hypothesis that LTH predicts outcome, because it is proportional to the energy that actually reaches the stone. As the intracorporeal portion of distance to the focal point increases, stone fragmentation and clearance is reduced. Other important drawback is that other variables that have been related to outcome, such as stone location and composition $(9,10)$, and data obtained from a NCCT such as radiographic density measured in Hounsfield Units (HU) (11-13), anatomical findings and SSD $(4,14)$ were not included in the analysis. A special consideration should be made regarding SSD, a test addresses the same issue as the Lithotripsy table height. Apparently shockwaves aren't conducted perfectly in human tissue, and if more tissue is interposed between the shockwave generator, less energy is likely to reach the stone and cause its fragmentation. Unfortunatel,y most patients in our study were preoperatively evaluated with KUB / US and in those who had a NCCT, SSD was not available, so it remains to be determined which test is more reliable as an outcome predictor as these haven't yet been compared. Theoretically, LTH could be more accurate because it is inversely proportional the distance from the stone to the skin where the shockwaves are actually entering the body -this distance is not fixed as human tissue is lax and can be compressed differently in the CT-scan table than when the therapy head is attached for SWL- whereas SSD on the CTscan is an average of three arbitrary measurements from the stone to the skin at 0,45 and 90 degrees and does not necessarily represent the area where the shockwaves are being conducted, for example for SWL in the prone position. Having this information preoperatively in clinic is certainly more ideal than obtaining it right before the procedure, but this issue can be discussed preoperatively and a different procedure (such as Retrograde Intra-Renal Surgery) could be performed in the same table if expected SWL success is very low. A question that arises from our study is if it's the ratio of tissue to water, or only the total amount of tissue within the conical area where the shockwaves are conducted -analogue to the SSD- that determines the amount of energy actually delivered to the stone. Lithotripters 
with a larger and broader parabola have larger focal distances, which varies the ratio of tissue to water without modifying the SSD. Unfortunately it is not possible to extrapolate the impact of different focal distances for different lithotripters, because our results were obtained using only one lithotripter, so the focal distance was the same for every procedure in this study. The comparison of lithotripters with different focal distances will help understand better the way shockwaves are conducted in the human tissue. We believe that even though a larger focal distance allows reaching 'deeper' stones, this patients will still present poorer outcomes than those with more superficial stones, meaning that the LTH effect will be present in every lithotripter but exact numbers may differ. Numerical data shown is only valid for the lithotripter used (Modulith SLX, Karl Storz, Germany), and, these findings need to be validated in studies comparing different machines.

\section{CONCLUSIONS}

SWL continues to be standard of care for many patients with stone disease, having satisfactory overall success rates. In this study, patients with residual fragments presented a significantly lower LTH than stone free patients. These findings could aid in patient selection, as an endourologic procedure might be performed instead, even in the same setting, for patients with very low LTH along with other variables predicting poor outcomes for SWL, and SWL could be considered for larger stones in patients with high LTH. Further studies are warranted to assess this variable in other lithotripters and consider other variables.

\section{ABBREVIATIONS USED}

LTH $=$ Lithotripsy Table Height; SWL $=$ Shockwave Lithotripsy; KUB = Kidney, ureters, bladder X-ray study; BMI = Body Mass Index; SSD = Skin-to-Stone Distance; HU = Hounsfield Units; NCCT $=$ Non Contrast-enhanced Computed Tomography; ROC $=$ Receiver Operating Characteristic; $\mathrm{SF}=$ Stone Free; $\mathrm{RF}=$ Residual Fragments; OR $=$ Odds Ratio; $\mathrm{SE}=$ Standard Error; $\mathrm{SD}=$ Standard Deviation; $\mathrm{CI}=$ Confidence Interval

\section{CONFLICT OF INTEREST}

None declared.

\section{REFERENCES}

1. Chaussy CG, Fuchs GJ: Current state and future developments of noninvasive treatment of human urinary stones with extracorporeal shock wave lithotripsy. J Urol. 1989; 141: 782-9.

2. Drach GW, Dretler S, Fair W, Finlayson B, Gillenwater J, Griffith D, et al.: Report of the United States cooperative study of extracorporeal shock wave lithotripsy. J Urol. 1986; 135: 1127-33.

3. Winfield HN, Clayman RV, Chaussy CG, Weyman PJ, Fuchs GJ, Lupu AN: Monotherapy of staghorn renal calculi: a comparative study between percutaneous nephrolithotomy and extracorporeal shock wave lithotripsy. J Urol. 1988; 139: 895-9.

4. Mezentsev VA: Extracorporeal shock wave lithotripsy in the treatment of renal pelvicalyceal stones in morbidly obese patients. Int Braz J Urol. 2005; 31: 105-10.

5. Smith AD, Badlani GH, Demetrius B, Clayman RV, Dacimo SG, Kavoussi LR, et al.: In: Cleveland RO, McAteer JA (ed.), The Physics of Schock Wave Lithotripsy. Smith's Textbook of Endourology. Quality medical publishing, 1996; pp. 317-32.

6. Shouman AM, Ziada AM, Ghoneim IA, Morsi HA: Extracorporeal shock wave lithotripsy monotherapy for renal stones $>25 \mathrm{~mm}$ in children. Urology. 2009; 74: 109-11.

7. Picramenos D, Deliveliotis C, Alexopoulou K, Makrichoritis C, Kostakopoulos A, Dimopoulos C: Extracorporeal shock wave lithotripsy for renal stones in children. Urol Int. 1996; 56: 86-9.

8. D'Addessi A, Bongiovanni L, Sasso F, Gulino G, Falabella R, Bassi P: Extracorporeal shockwave lithotripsy in pediatrics. J Endourol. 2008; 22: 1-12.

9. Tiselius HG, Alken P, Buck C, Gallucci M, Kanoll T, Sarica K, et al.: Guidelines on Urolithiasis. Arnhem. European Association of Urology (EAU). 2008; p. 128.

10. Preminger GM, Tiselius HG, Assimos DG, Alken P, Buck AC, Gallucci M, et al.: 2007 Guideline for the management of ureteral calculi. Eur Urol. 2007; 52: 1610-31.

11. Krishnamurthy MS, Ferucci PG, Sankey N, Chandhoke PS: Is stone radiodensity a useful parameter for predicting outcome of extracorporeal shockwave litho- 
tripsy for stones $<$ or $=2 \mathrm{~cm}$ ? Int Braz J Urol. 2005; 31 : 3-8; discussion 9.

12. El-Nahas AR, El-Assmy AM, Mansour O, Sheir KZ: A prospective multivariate analysis of factors predicting stone disintegration by extracorporeal shock wave lithotripsy: the value of high-resolution noncontrast computed tomography. Eur Urol. 2007; 51: 1688-93; discussion 1693-4.

13. Pareek G, Hedican SP, Lee FT Jr, Nakada SY: Shock wave lithotripsy success determined by skin-to-stone distance on computed tomography. Urology. 2005; 66: 941-4.

14. Robert M, A'Ch S, Lanfrey P, Guiter J, Navratil H: Piezoelectric shockwave lithotripsy of urinary calculi: comparative study of stone depth in kidney and ureter treatments. J Endourol. 1999; 13: 699-703.

Accepted after revision:

October 17, 2010

\author{
Correspondence address: \\ Dr. Pedro Recabal \\ Department of Urology \\ Hospital Clínico Universidad de Chile \\ Santos Dumont 999, Independencia \\ Santiago, Chile \\ Fax: + 552 777-8758 \\ E-mail: pedrorecabal@gmail.com
}

\section{EDITORIAL COMMENT}

Extracorporeal shock wave lithotripsy (SWL) has remained the preferred treatment for small to moderate sized kidney stones. Stone size, location, composition as well as patient characteristics (e.g. body mass index, BMI) all influence outcome. Sampaio and Aragao (1) first described three anatomical factors that influence the success of fragment clearance after ESWL, including an infundibulopelvic angle of $>90^{\circ}$, an infundibular width of $>4$ $\mathrm{mm}$ and special arrangement of the lower pole calyceal group. Pareek et al. (2) evaluated 64 patients with stones of 5-15 mm and found a statistically significant association between the stone-free rate, stone density and BMI (measured as skin-to-stone distance on CT) The most powerful predictor of failure was a skin-to-stone distance of $>8 \mathrm{~cm}$.

In this study the authors analysed stone size, BMI and lithotripsy table height (LTH) as independent predictors of outcome following SWL. All procedures were performed with the Modulith SLX lithotripter. 56 patients were prospectively analysed. The main endpoint of this manuscript was that LTH was the only independent predictor of treatment results. Patients with LTH $<218$ had much higher risk of residual stones than patients with LTH $>218$.

The aperture focus distance varies with different types of lithotripter. The European Guidelines on Urolithiasis (3) recommends calculating the optimal (or maximal) skin-to-stone distance for each type of lithotripter. To my knowledge, this was the first report that offered LTH to help improve patient selection for SWL. The results were encouraging. I feel LTH calculation prior SWL could be recommended in obese patients.

\section{REFERENCES}

1. Sampaio FJ, Aragao AH: Limitations of extracorporeal shockwave lithotripsy for lower caliceal stones: anatomic insight. J Endourol. 1994; 8: 2417.

2. Pareek G, Schluckebier J, Hedican S, Lee F Jr, Nakada S. Measurement of skin to stone distance using non-contrast computed tomography can predict outcome following shockwave lithotripsy of lower pole renal stones. Program and abstracts of the American Urological Association Annual Meeting; 2005; May 21-6. San Antonio, Texas. Abstract\# 1519.

3. Türk CH, Knoll T, Petrik A, Sarica K, Seitz C, Straub M, et al.: Guidelines on Urolithiasis. Eur Urol. 2010, 251-282

Dr. V.A. Mezentsev Harrogate and District NHS Foundation Trust Yorkshire Deanery, 19 Old Mill View Dewsbury, WF12 9QJ, United Kingdom E-mail:vitalimezentsev@hotmail.com 